\title{
Hydromorphone attenuates intercellular adhesion molecule-1 expressions induced by lipopolysaccharide on HCT-116 human colon cancer cells
}

\author{
Jae Jin Lee, Woon Young Kim, Ji Hye Um, and Too Jae Min \\ Department of Anesthesiology and Pain Medicine, Korea University Ansan Hospital, Ansan, Korea
}

Resently, Min et al. [1] showed that morphine attenuated the stimulant effect of lipopolysaccharide (LPS). LPS is especially abundant in the colon. LPS stimulates the synthesis and secretion of various cytokines and other pro-inflammatory mediators such as interleukin-1 $\beta$ (IL-1 $\beta$ ), IL-8, IL-6, and vascular endothelial growth factor [2]. These cytokines induced endothelial adhesion molecule expressions through a nuclear factor $\kappa \mathrm{B}(\mathrm{NF}-\kappa \mathrm{B})$ dependent pathway. LPS has been shown to be a stimulant of vascular metastatic processes as well,especially in colon cancer. Min et al. [1] also demonstrated that the expression of adhesion molecules such as intercellular adhesion molecule-1 (ICAM1), vascular cell adhesion molecule-1 (VCAM-1), and E-selectin were attenuated on human umbilical vascular endothelial cells (HUVECs) when LPS was co-administered with morphine. However, the adhesion ratio of cancer cells to HUVECs was not demonstrated at that time. Hydromorphone is a semi-synthetic opioid that is a hydrogenated ketone of morphine. Hydromorphone was selected in this study because it had a longer duration of action and less intense adverse effects such as pruritus and nausea than morphine $[3,4]$. Due to these potential advantages, hydromorphone is widely used in cancer patients for relief of pain. Therefore, in this study, we investigated the adhesion ratio of colon cancer cells to LPS-stimulated HUVECs and whether co-administration with hydromorphone attenuated the adhesion of colon cancer cells to HUVECs.

Four different growth conditions were used in this study: (1) HUVECs incubated in endothelial growth medium without growth factors (the control group), (2) HUVECs incubated with the supernatant from LPS-stimulated HCT 116 cells (the LPS-SUP group), (3) HUVECs incubated with the supernatant from HCT 116 cells that were not stimulated with LPS (the SUP group), and (4) hydromorphone co-administered to HCT 116 cells during stimulation with LPS (the HYDRO + LPS-SUP group). The LPS-SUP, the HYDRO + LPS-SUP groups were prepared following a previously published experimental protocol (Fig. 1A) [1]. Hydromorphone was supplemented at three different concentrations, $0.1,1$, and $10 \mu \mathrm{M}$ [5]. The surface expression of adhesion molecules was measured by flow cytometry (Facsflow from BD Biosciences, San Jose, CA, USA) by using an ICAM-1 specific fluorescein isothiocyanate-labeled antibody. And we demonstrated that the adhesion ratios of HCT 116 cancer cells to HUVECs by using carboxy-flourescein HCT 116 cancer cells. Adhesions with HUVECs were counted by optical microscopy. As result, the adhesion ratios of HCT 116 cancer cells to HUVECs were significantly lower in the HYDRO + LPSSUP group than in the other groups (Fig. 1B). Therefore, we concluded hydromorphone might reduce the adhesion of cancer cells to the vascular wall by reducing the expression of adhesion molecules on endothelial cells.

In this study, we found evidence, which suggests that hydromorphone may have anti-tumor effects similar to morphine. Considering the benefits of hydromorphone over morphine, these results show that hydromorphone might be the opioid analog of choice for treatment of cancer pain. Although this study

Corresponding author: Too Jae Min, M.D., Ph.D., Department of Anesthesiology and Pain Medicine, Korea University Ansan Hospital, 123, Jeokgeum-ro, Danwon-gu, Ansan 425-707, Korea. Tel: 82-31-412-5488, Fax: 82-31-412-5294, E-mail: minware2@lycos.co.kr

(c) This is an open-access article distributed under the terms of the Creative Commons Attribution Non-Commercial License (http:// creativecommons.org/licenses/by-nc/3.0/), which permits unrestricted non-commercial use, distribution, and reproduction in any medium, provided the original work is properly cited. 
has the limitation of being an in vitro analysis, we concluded that hydromorphone might reduce LPS-stimulated metastasis in colon cancer by attenuating the expression of ICAM-1 on endo- thelial cells. In the future, we plan to evaluate the anti-metastatic effect of hydromorphone using both in vivo and in clinical approaches.

A

Experimental protocol

$24 \mathrm{hr}$

Control group

Supernatant (EGM)

HUVEC

LPS-stimlated supernatant group (LPS-sup group)

Hydromorphone co-treated with LPS

\section{Media was changed}

\begin{tabular}{|c|c|c|}
\hline $4 \mathrm{hr}$ & $24 \mathrm{hr}$ & \multirow{2}{*}{$\begin{array}{c}24 \mathrm{hr} \\
\text { Supernatant (EGM) }\end{array}$} \\
\hline RPMI + LPS & EGM & \\
\hline \multicolumn{2}{|c|}{ HCT 116 cell line } & HUVEC \\
\hline
\end{tabular}

Medium was changed

\begin{tabular}{|c|c|c|}
\hline $4 \mathrm{hr}$ & $24 \mathrm{hr}$ & $24 \mathrm{hr}$ \\
\hline RPMI + LPS & EGM & Supernatant (EGM) \\
\hline
\end{tabular}

Hydromorphone Media of HUVEC was replaced by added supernatant of HCT 116 cell line

B
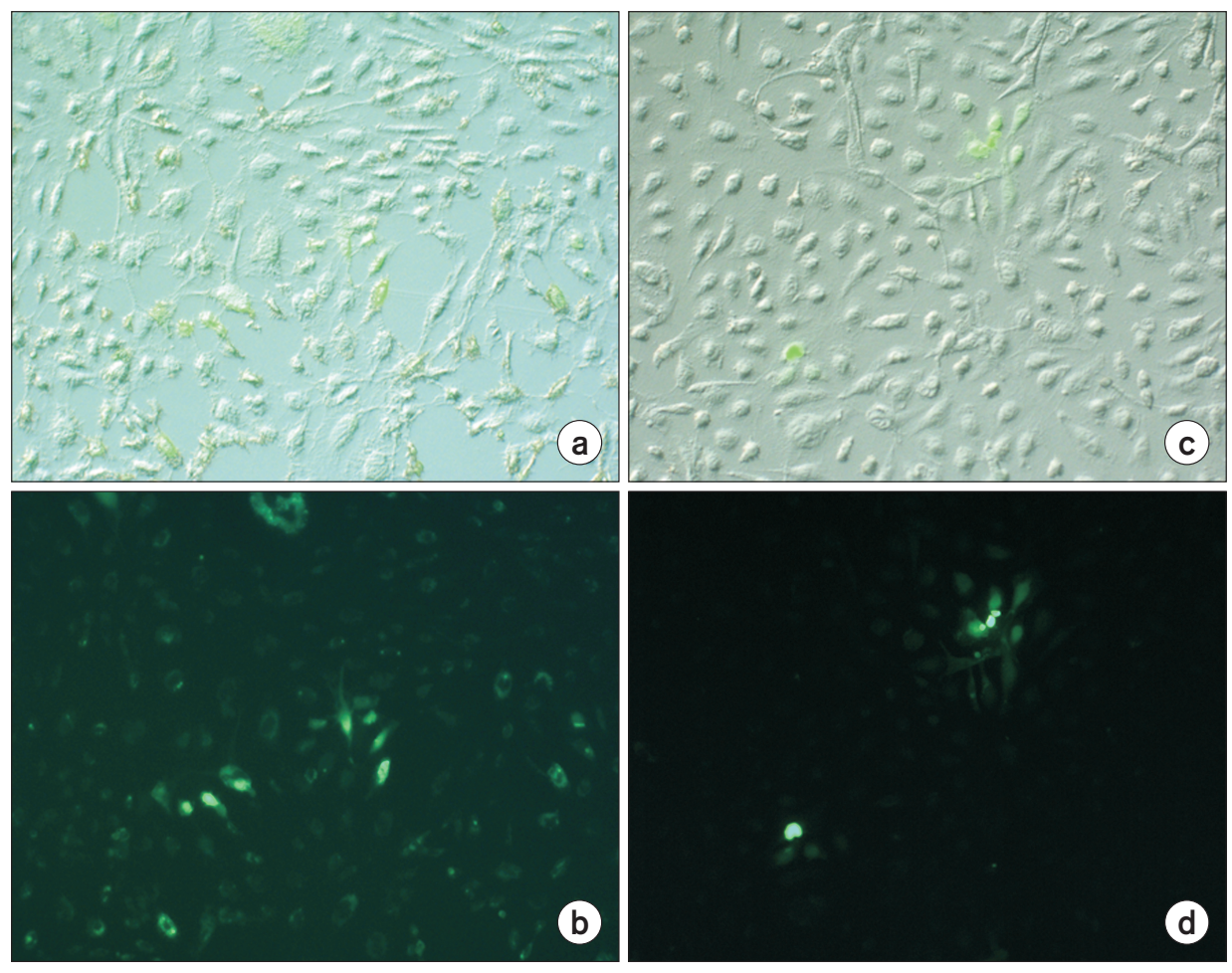

Fig. 1. (A) Experimental protocol. For the control group, HUVECs were incubated in growth factor-free endothelial medium. For the LPS-Sup group, HUVECs were incubated with the supernatant of LPSstimulated human HCT 116 colon carcinoma cells. For the HYDRO + LPS-SUP group; hydromorphone was added to the LPS-supernanant prior to incubation with HUVECs. Endothelial growth medium is an endothelial cell growth medium without endothelial growth hormone. (B) Ratios of adhesion of HCT 116 human carcinoma cells to HUVECs. The adhesion ratios for HCT 116 cells to HUVECs were measured after $4 \mathrm{~h}$ of incubation. The values are the mean \pm SD of 25 experiments. $* \mathrm{P}<0.05$ vs. the LPS-SUP group. 


\section{References}

1. Min TJ, Park SH, Ji YH, Lee YS, Kim TW, Kim JH, et al. Morphine attenuates endothelial cell adhesion molecules induced by the supernatant of LPS-stimulated colon cancer cells. J Korean Med Sci 2011; 26: 747-52.

2. Smirnova MG, Guo L, Birchall JP, Pearson JP. LPS up-regulates mucin and cytokine mRNA expression and stimulates mucin and cytokine secretion in goblet cells. Cell Immunol 2003; 221: 42-9.

3. Shulman MS, Wakerlin G, Yamaguchi L, Brodsky JB. Experience with epidural hydromorphone for post-thoracotomy pain relief. Anesth Analg 1987; 66: 1331-3.

4. Rapp SE, Egan KJ, Ross BK, Wild LM, Terman GW, Ching JM. A multidimensional comparison of morphine and hydromorphone patientcontrolled analgesia. Anesth Analg 1996; 82: 1043-8.

5. Borowitz JL, Gunasekar PG, Isom GE. Hydrogen cyanide generation by mu-opiate receptor activation: possible neuromodulatory role of endogenous cyanide. Brain Res 1997; 768: 294-300. 